\title{
Reversible response of a Magnetic Surface Acoustic Wave device with perpendicular magnetization
}

\author{
Vincent Polewczyk, ${ }^{1}$ Daniel Lacour, ${ }^{1}$ Karine Dumesnil, ${ }^{1}$ Hamid Mjahed, ${ }^{1}$ \\ Sébastien Petit-Watelot, ${ }^{1}$ François Montaigne, ${ }^{1}$ Omar Elmazria ${ }^{1}$ and Michel \\ Hehn ${ }^{1}$ \\ ${ }^{1}$ Institut Jean Lamour (UMR 7198), CNRS-Université de Lorraine, 54000 Nancy, France \\ E-mail: vincent.polewczyk@gmail.com
}

\begin{abstract}
Magnetic Surface Acoustic Wave (MSAW) devices combine a magnetostrictive material with a piezoelectric material to enable the sensing/measurement of magnetic fields by electrically generated SAWs. In such systems, the response is strongly dependent on the field direction with respect to the magnetic anisotropy of the magnetic material, which could give rise to detrimental hysteretic behaviour. The challenge in the current study is to control the shape of MSAW responses and remove the hysteresis in the magneto-acoustic response. We report especially on the magnetic response of a MSAW device with perpendicular magnetic anisotropy, rarely reported in literature. On the basis of the MSAW response with in-plane magnetic anisotropy studied previously, the MSAW signal with out-of-plane magnetization could be understood for all the field configurations. The final response is hysteresis free, which is suitable for interrogation of out of the plane magnetic fields. Such a technology could therefore complete the "in-plane" sensing technology to build up 3D sensors.
\end{abstract}

Keywords: SAW, Surface acoustic wave, Magnetization, Magnetic anisotropy, Magneto-acoustic, Piezoelectric, Resonator.

\section{Introduction}

From the end of the seventies, magnetic surface acoustic wave systems have been proposed, based on the strain induced by a magnetostrictive materials [1-19]. Most of the research activities appear to focus on two main aspects: (i) the modification of the SAW characteristics (resonance, shape ...) by an external static magnetic field [1-11] (ii) and the elastic excitation of the magnetization dynamics, i.e. SAW FerroMagnetic Resonance (SAW-FMR) [12-19]. These studies are respectively pursued for magnetic sensing and for magnetic device applications. In both cases, the MSAW responses are correlated either to the magnetic properties of the thin film deposited in the gap between input and output InterDigital Transducers (IDT) or on top of the SAW resonator, or to the magnetic properties of the magnetic IDTs [7]. The use of a magnetic material, usually with anisotropic properties, could be responsible for hysteresis in the MSAW response that either can bring additional features to the device (bipolar response of sensors for example [7]) or can be detrimental to a univocal MSAW frequency vs applied field response. The sensing interrogation is usually reported for inplane applied magnetic field [5-8]. Along the easy axis of magnetization, the MSAW response is hysteretic while it can be hysteresis free along the hard axis of magnetization. In such systems, the SAW frequency variation for out of plane fields is either close to zero $[3,4]$ or hysteretic. To envision the development of out of the plane magnetic sensors, i.e. to measure a field perpendicular to the sensor plane, one must carefully design the magnetic characteristics, especially the magnetic anisotropy, to get rid of such non-reversible behavior. This prerequisite would pave the way to $3 \mathrm{D}$ magnetic detection in a single device. 
In this paper, we report on MSAW devices where IDTs are patterned from a $200 \mathrm{~nm}$ thick textured $\mathrm{Ni}$ layer, and in a resonator geometry for future wireless measurements. The piezoelectric substrate is $\mathrm{LiNbO}_{3}$ (LNO) in two different orientations: LNO $41 Y$ and LNO $128 Y$-cuts. Similar results were found for both orientations, thus only those obtained on LNO $41 Y$ are reported in the following, with a specific focus on Rayleigh Waves (RW). A complete description of the SAW geometry can be found in [20]. We present here typical results obtained on a specific device, but multiple devices were patterned and analyzed, which could confirm the reproducibility of the main characteristics.

\section{Characteristics of the pristine film}

The multilayer stack used in this study has been grown by UHV sputtering. In a first step, a $5 \mathrm{~nm}$ thick Tantalum buffer layer is deposited on LNO in order to improve the adhesion of the Nickel layer and also to promote a (111) texture. It is well known that, in standard conditions, sputtered Ta with a thickness smaller than $5 \mathrm{~nm}$ is amorphous and is used to promote the subsequent growth of (111) fcc closed packed planes [21]. A $200 \mathrm{~nm}$ thick Ni layer is then deposited and eventually capped with a $5 \mathrm{~nm}$ thick Pt layer to protect the $\mathrm{Ni}$ from contamination and oxidation. $\mathrm{Ni}$ is deposited using an RF Ar plasma with a power of $90 \mathrm{~W}$ and an Ar pressure of $7 \times 10^{-3}$ Torr. In these conditions, the deposition rate is 0.1 $\mathrm{nm} . \mathrm{s}^{-1}$. The sample-holder is moved above the Ni target (forth and back along one axis) during the process, to insure a good homogeneity of the deposited layer.

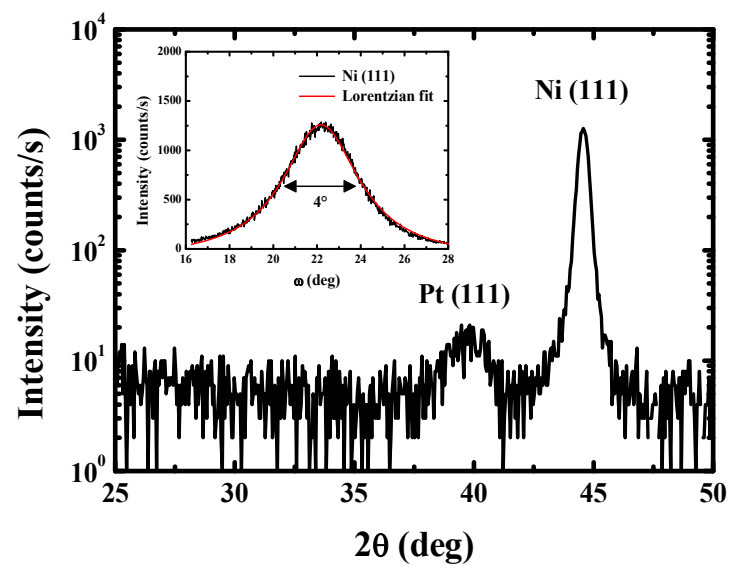

Figure 1. Large angle specular X-ray measurement performed for a $200 \mathrm{~nm}$ thick Ni film deposited on LNO 41 Y-cut. Inset: rocking curve across the (111) Ni Bragg peak.

Large angle X-ray scattering measurements performed for a $\mathrm{Ni}$ film deposited on LNO $41 \mathrm{Y}$-cut is presented in figure 1. Since the alignment is performed with respect to the sample surface, the LNO substrate doesn't give rise to any specific Bragg peak in this specular configuration [20].

This measurement shows the occurrence of a small contribution due to the $\mathrm{Pt}$ capping and of the Bragg peak associated to $\mathrm{Ni}$ (111) planes. Since complementary experiments in asymmetric geometry did not reveal any other Bragg peaks at specific azimuthal angle, we conclude to a polycrystalline order with a texture along [111]. The rocking curve measured across this Bragg peak presents a Full Width at Half Maximum of approximately $4^{\circ}$. The angular position of the (111) Bragg peak indicates a compressive strain along the growth direction of approximatively $-0.26 \%$. As expected, no evidence of texture could be found for the Ta layer.

Hysteresis loops have been measured with the magnetic field applied in-plane (IP) along LNO X and $\mathrm{LNO} \mathrm{X}+90^{\circ}$ directions (figure 2 (a)) and with the magnetic field applied perpendicular to the plane (OOP) (figure 2 (b)).

One can first underline that the saturation magnetization value is close to the value expected from bulk Ni (490 kA.m ${ }^{-1}$ [22]). The OOP curve (figure 2 (b) green) is typical of a perpendicular magnetic anisotropy with strong stripes domains configurations, as revealed by the typical opening of small hysteresis loops close to the saturation [23-28]. In this configuration, no remanence for the longitudinal component of magnetization is expected since domains with magnetization pointing up or down along the OOP direction compensate each other.

The stripes domains configuration in magnetic materials with perpendicular magnetic anisotropy occurs above a critical thickness to save part of the perpendicular anisotropy energy and reduce the demagnetizing field. Such a transition is expected when the thickness of a Ni layer increases from 50 $\mathrm{nm}$, for which an in-plane magnetization is observed, to 200 $\mathrm{nm}[20]$. This particular state of $\mathrm{Ni}$ is reported in literature [29, 30 ] and could be stabilized by a supplementary magnetoelastic energy contribution in the case of a perpendicular compressive state [28].

Since the Ni layer is textured in the direction perpendicular to film plane, the IP behavior is expected to be isotropic, as observed in figure 2 (a). The large remanence and coercivity for IP loops is then attributed to the magnetization in center of the domain walls, mainly aligned along the saturating field direction [23-27].

\section{Micro-processing of the device and first characterizations (RF \& magnetic)}

The resonator geometry, where a single antenna could be connected to the single IDT system that plays the role of receiver and emitter, is chosen to allow future wireless measurements (see figure 3 ). The sensor structure is designed using a synchronous single-port SAW resonator. The wavelength $(\lambda)$ and the metallization ratio are equal to $6.5 \mu \mathrm{m}$ and $50 \%$ respectively. Each finger is $1.625 \mu \mathrm{m}$ wide and 270 $\mu \mathrm{m}$ long. The number of finger pairs is 100 in the resonator part while the reflectors are composed of 200 fingers. The 

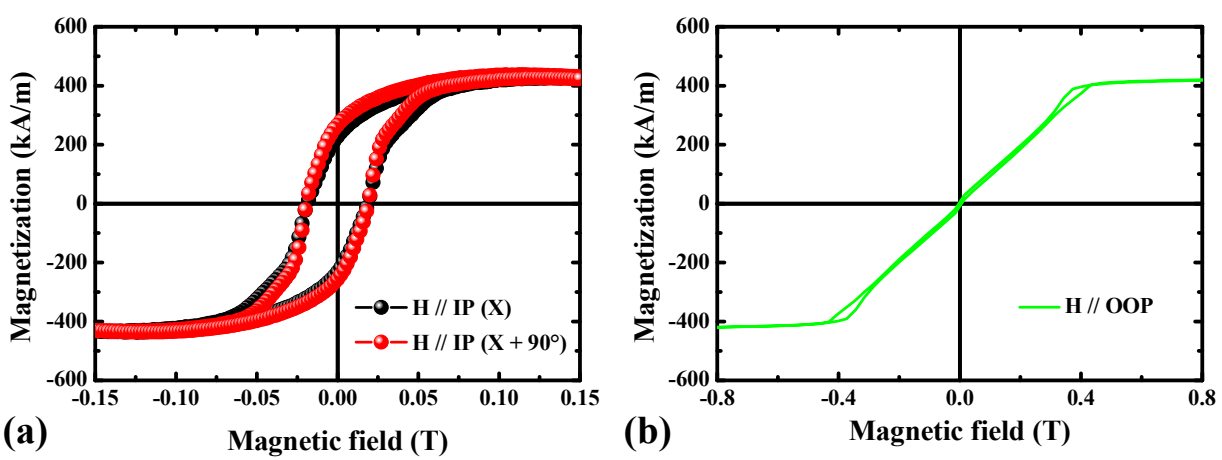

Figure 2. Room temperature hysteresis loops measured for a $200 \mathrm{~nm}$ thick Ni film deposited on LNO 41 Y-cut with the magnetic field applied along two in-plane directions (a) and perpendicular to the sample plane (b).

IDTs are patterned from the continuous film using optical lithography and dry Ar ion etching. After film deposition, a positive resist is spin-coated on the sample surface.

After insolation through an optical mask, the SAW shape is transferred to the photoresist layer. This pattern is subsequently transferred to the magnetic layer using dry Ar ion etching. The etching process is followed in-situ using second ion mass spectrometry such that the etching is stopped in the LNO substrate. The orientation of the IDTs is such that the generated acoustic waves propagate along the $\mathrm{X}$ crystallographic axis of the LNO 41Y-cut substrate.

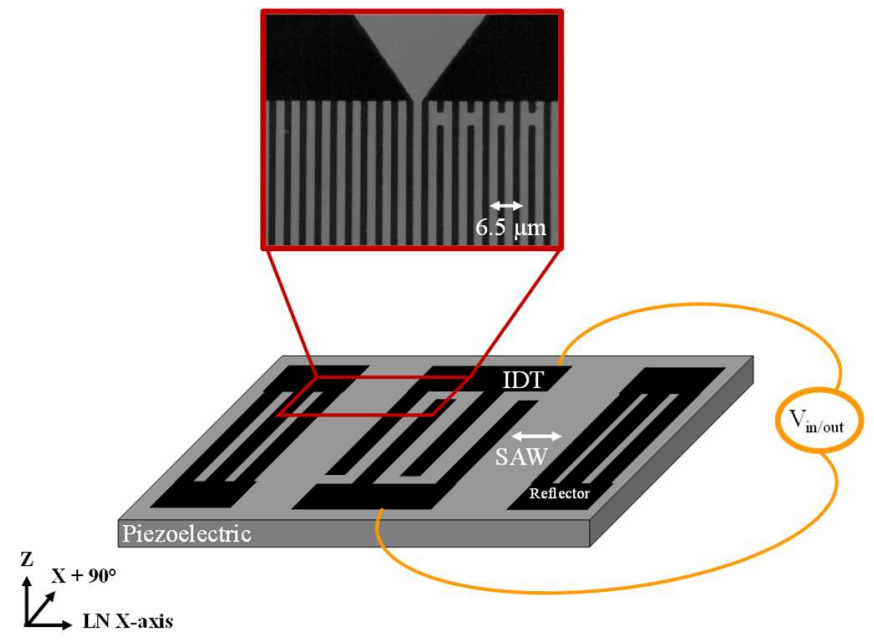

Figure 3. Sketch and optic microscope view of the resonator geometry for magnetic $S A W$ devices.

The magnetic and piezoelectric materials are presented respectively in black and grey. The arrow represents the direction of wave propagation. A single IDT constitutes the acoustic wave emitter and receiver. $\left(X, X+90^{\circ}, Z\right)$ is the $L N O$ coordinate basis with the $X$-axis parallel to the $L N O X$-axis.

The frequency response of the resonator is measured using a network analyzer (Agilent PNA 5230A, Santa Clara, CA) and a RF probe station (PM5, Suss Microtec). For all measurements presented here, the intensity of the VNA transmitted signal is fixed to $0 \mathrm{~dB}$. It was checked that the shape of the response does not evolve as a function of intensity, i.e., that the device is working in its linear regime. The output $\mathrm{S}_{11}$ signal of the MSAW device without applied magnetic field is presented in figure 4. The resonance frequency (Fr) is equal to $552 \mathrm{MHz}$ and is in good agreement with the predictions considering the acoustic velocity of

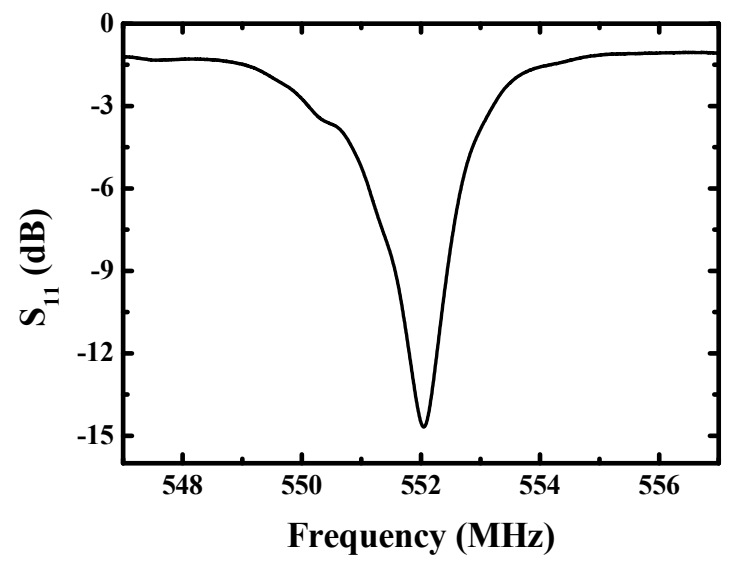

Figure 4. $S_{11}$ signal versus frequency for a $200 \mathrm{~nm}$ thick Nickel-based device deposited on LNO 41 Y-cut.

Rayleigh wave ( $\mathrm{Vr}$ ) along the $\mathrm{X}$ direction of the LNO 41Y-cut substrate [31] and the acoustic wavelength $(\mathrm{Fr}=\mathrm{Vr} / \lambda)$. Rayleigh Waves (RW) are surface acoustic wave that arise from the interaction of acoustic waves with the interface and penetrate the host material less than a few acoustic wavelengths [32]. RW result from the superposition of shear vertical and longitudinal atomic displacements that define an elliptical movement.

While the hysteresis loop gives a hint to extrapolate the existence of stripes domains in the pristine film, a direct observation of the stripes domains state in the IDT after technological process is mandatory. An Atomic Force Microscopy - Magnetic Force Microscopy (AFM-MFM) study was thus carried out. Figure 5 ( $a$ and $b$ ) provides the AFM image of the surface, and the topography profile along the red double arrows. Measurements were carried out in tapping and lift modes for AFM-MFM respectively. The lift height was fixed to $50 \mathrm{~nm}$. The measurement of the MFM contrast is reported in figure 5 

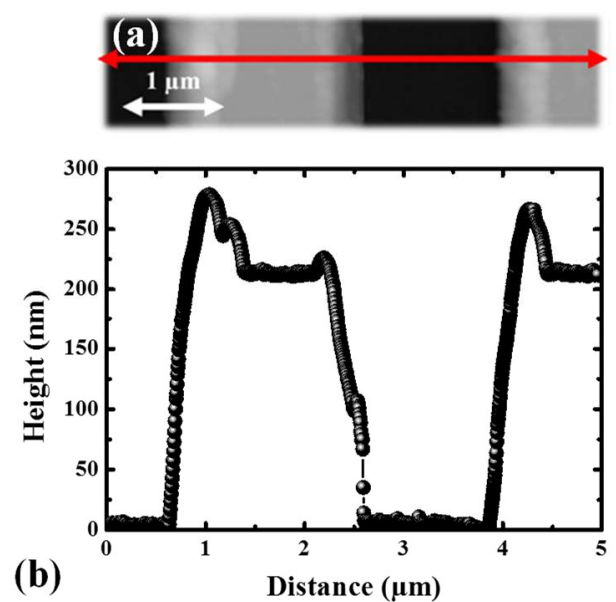

Figure 3. (a) AFM image of fingers patterned from a $200 \mathrm{~nm}$ thick Nickel film deposited on LNO $41 Y$-cut with a wavelength of $6.5 \mu \mathrm{m}$ and a metallization ratio of $50 \%$. (b) Associated profile along the finger width (along the red double arrows in (a)). (c) MFM image for the same fingers and for zero applied field after application of an in-plane saturating magnetic field along the finger's width. (d) Associated profile (along the red double arrows in (c)) of the stripe domains structure.

(c). It shows the phase contrast for zero applied field after application of an IP saturating field, perpendicular to the finger's length. The profile along the red double arrows is given in figure $5(\mathrm{~d})$.

As observed in figure $5(\mathrm{~b})$, the effective width of the fingers $(1.70 \mu \mathrm{m})$ exceeds the nominal one $(1.625 \mu \mathrm{m})$, with the occurrence of edge parts of larger thickness, up to $275 \mathrm{~nm}$. Those so-called "rabbit ears" (approx. $100 \mathrm{~nm}$ wide) appear after the etching process to define the IDT's fingers and most likely originate from the redeposition of sputtered atoms during the Ar dry etching process. Since the redeposited atoms are magnetic, these ears should present some magnetic contrast and make the MFM observation more difficult.

The MFM signal is proportional to the second derivative of the stray field along the direction perpendicular to film plane when the tip is magnetized in the same direction. While this contrast is mainly located close to areas where the magnetization is strongly inhomogeneous (domain walls for example), in the case of a domain structure with perpendicular magnetization and domain size of the order of $100 \mathrm{~nm}$, the contrast is centered on the domains [27]. As a result, with the support of the magnetic hysteresis loop, the MFM profile in figure 5 (d) confirms the presence of magnetic domains with alternately up and down orientation, and a period of approximatively $300 \mathrm{~nm}$. As previously observed in other materials, applying a saturating in-plane field leads then to a remanent state with a mixture of bubbles and stripes mainly orientated along the initial field direction [23-27]. The resulting configuration is here more complex because of the reduced lateral width of the IDT and the presence of the "rabbit ears". The MFM contrast in the rabbit ears is difficult to understand. As these edges' thickness is larger than the thickness of the Nickel layer, we can admit that in this region, the magnetization is also perpendicular. Since the period of the magnetic stripes varies as the square-root of the film thickness [23-27], the lateral period should be around $350 \mathrm{~nm}$ in those regions.

\section{Field dependence of the $\mathbf{R W}$ frequency}

In order to record the Rayleigh Wave frequency as a function of the applied magnetic field, the resonator is bonded to a copper-coated Printing Circuit Board (PCB) suitable for microelectronics applications. Connections are established between the PCB and SAW device using wire bonding (20$\mu \mathrm{m}$-diameter gold wire). $\mathrm{PCB}$ is connected to a network analyzer via RF cables and non-magnetic SMA connector The whole stack is then placed between the coils of an electromagnet.

The RW frequency variations versus field are presented in figure 6 for fields applied along in-plane LNO X (black) and LNO X $+90^{\circ}$ (red) directions, and along the perpendicular-tothe-plane (green) direction. The magnetic field is swept till a magnetic value in accordance with the magnetic hysteresis loops recorded in Figure 2. Figure 6 (b) is a zoom of the central area, around the zero applied field.

When the field is applied along $\mathrm{LNO} \mathrm{X}+90^{\circ}$, i.e. along the IDT (red), the curve is typical of the behavior observed along the easy magnetization axis (EA) in $50 \mathrm{~nm}$ thick Ni based devices [20]. One can observe the occurrence of (i) the hysteresis due to the reversal of the in-plane magnetization component related to the shape of the fingers (ii) dynamical 

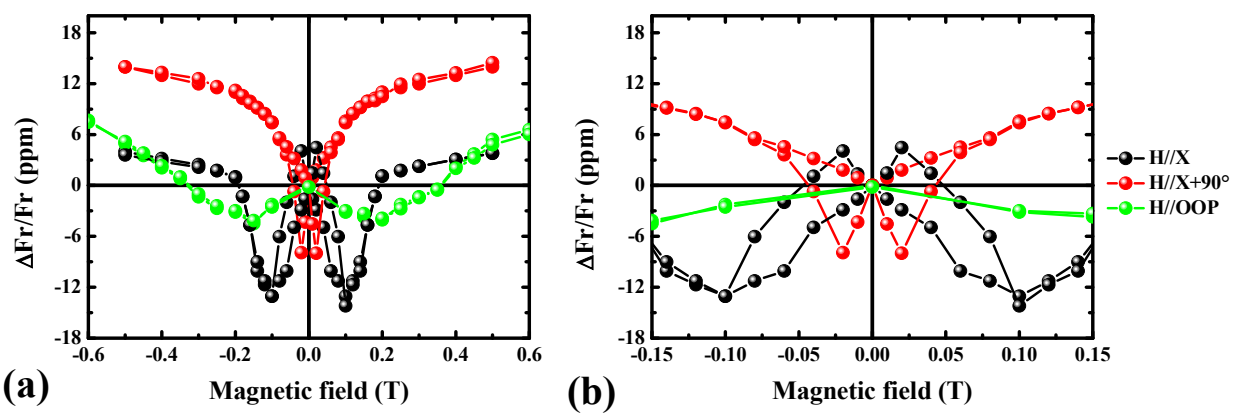

Figure 4. (a) Variation of the $R W$ frequency in the MSAW device, with the magnetic field applied in-plane along LNO X (black) and LNO X+90 (red), and out-of-plane (green). (b) Zoom of the central area, close to zero applied field.

effects, i.e. the effect on the SAW response of magnetization dynamics induced by the AC magnetoelastic field. Dynamical effects occur over the entire field range and are especially responsible for the field dependence existing beyond the magnetic saturation.

Such a response is consistent with the micromagnetic configurations (thin black arrows) depicted in figure 7 (a), as expected for the variation versus field of a stripe domains structure in an elongated finger [23-27]. From in-plane saturation along the finger length, the magnetization reorients perpendicular to film plane with up and down domains, giving rise to magnetic domain walls in which the magnetization is mainly oriented towards the initial saturation field direction. This orientation for net magnetization in domain walls is reinforced by the shape of the IDT fingers, favoring magnetization along the length of the structure. The rotation out of the field direction doesn't generate any hysteretic behavior. However, when the field is reversed, the magnetization inside the domain walls switches, leading to the appearance of an hysteresis [20]. This reversal occurs at \pm 25 $\mathrm{mT}$, close to the coercive field of the full film.

The occurrence a dynamical magnetoelastic field in this configuration where the magnetization remains in a plane perpendicular to the propagation direction is likely due to the presence of shear strains, as presented in the paper by Dreher et al. [33].

When the in-plane field is applied perpendicular to the IDT length (black curve), the response exhibits a "w" shape, typical of the behavior previously observed along a hard magnetization axis (HA) and related to the variation of the $\mathrm{C}^{\prime}{ }_{11}$ elastic constant [29]. A difference is however the opening of hysteretic parts in the $[-0.1 \mathrm{~T} /+0.1 \mathrm{~T}]$ field range, in agreement with the measurement of magnetization hysteresis loops (figure 2 (a)).

Top view of IDT's finger

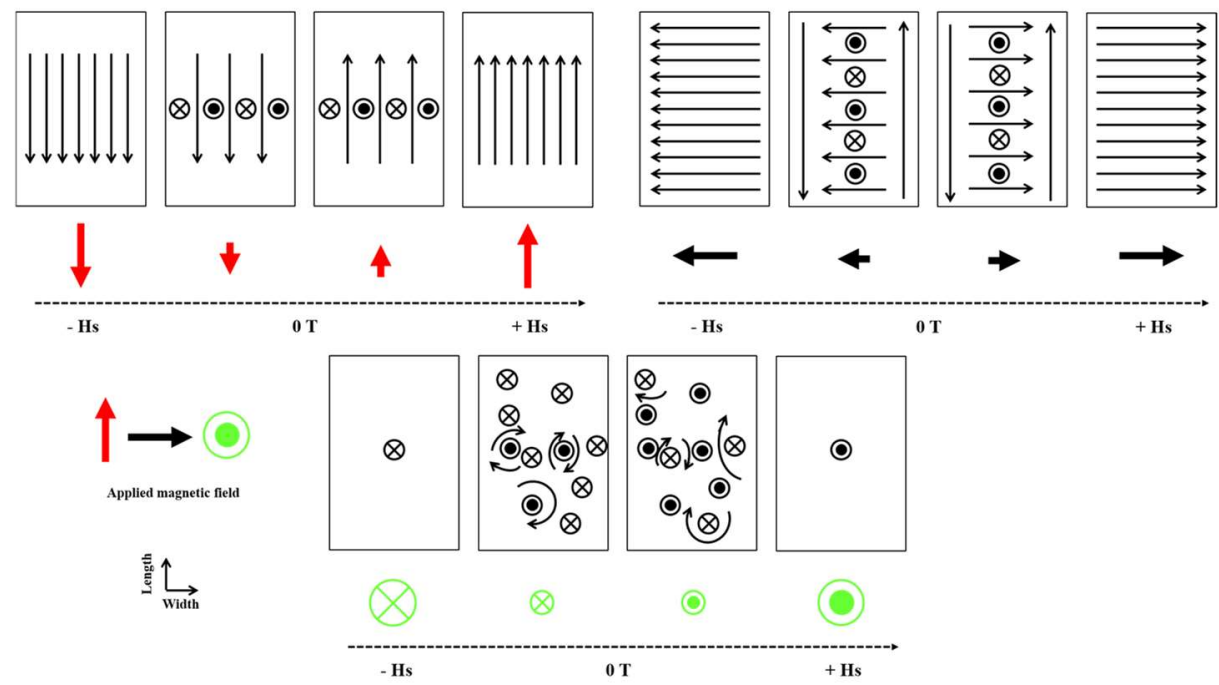

Figure 5. Sketch of the expected magnetic configurations as a function of the in-plane applied field along the IDT (a), perpendicular to the IDT (b) and of the field applied perpendicular to the IDT plane (c). Black and red arrows and green circles represent the direction of applied field. The length/diameter of arrows/circles are related to the field intensity. Black thin arrows represent the magnetization in the IDT finger. 
As for the LNO $\mathrm{X}+90^{\circ}$ direction, reducing the field from saturation drives the magnetization reorientation perpendicular to film plane, with alternately up and down magnetized domains as schematized in figure 7 (b). However, close to the edges, and with some extension in the core of the IDT, the magnetization is expected to be parallel to the IDT because of magnetostatic effects. The shape anisotropy is responsible for the increase of the field for which $\Delta \mathrm{Fr}$ is minimum and for the magneto acoustic saturation field, i.e. the field above which the resonance frequency varies slightly with field (see figure 6). In the case of a $200 \mathrm{~nm}$ thick Ni finger in a $6.5 \mu \mathrm{m}$ wavelength device $\left(\mathrm{Ms}=430 \mathrm{kA} \cdot \mathrm{m}^{-1}, 1.625 \mu \mathrm{m}\right.$ wide, $270 \mu \mathrm{m}$ long), one can calculate a demagnetizing field of approximatively $90 \mathrm{mT}$ along the finger width.

The hysteretic behavior observed for this in-plane field direction while the device exhibits perpendicular anisotropy is most likely linked to the switch of net magnetization in domain walls (thin black arrows between up and down domains in figure 7 (b)) when reversing the magnetic field.

For OOP applied fields (green curve), the MSAW response is reversible with the " $w$ " shape generally encountered when the field is applied along an in-plane HA. This is in strong contrast with Ni based devices with IP easy magnetization, where the OOP response is highly hysteretic, due to the hysteretic reversal of in-plane magnetization components [7].

The reversible OOP MSAW response in this $200 \mathrm{~nm}$ thick Nickel-based device is explained by the successive magnetic configurations sketched in figure 7 (c). At saturation (Hs), the magnetization is fully aligned along the perpendicular direction. When the applied field decreases, and for applied fields less than $400 \mathrm{mT}$, the IDT hosts first magnetic bubbles and then magnetic stripes, i.e. domain structures with up and down magnetization. Those domains are separated by Bloch walls with in-plane magnetization components that are, for perpendicular applied field, homogeneously distributed in the plane, as reported in [19-23]. In contrast to the cases in figure 7 ( $a$ and b), the wall contributions (thin black arrows in 7 (c)) are distributed in all in-plane directions since the in-plane symmetry is not broken. Such an isotropic distribution of inplane magnetization evolves for reversed field and eventually annihilates in a reversible way. This behavior therefore doesn't give rise to any hysteresis in the MSAW response. Neither an easy axis nor a hard axis response is expected. The magneto acoustic response is likely a combination of both contributions with (i) static effects due to the variation of the elastic constant and (ii) dynamical effects for parts of the domain walls with magnetization of relevant orientation respect to the acoustic wave propagation [33].

One can notice that the field sensitivity of this sensing device is relatively low, indeed smaller than what can be found for in-plane applied magnetic field interrogation in literature. If one considers the reversible field range between the remanence and the minimum of the curve along an IP hard axis, the sensitivity in our device is close to $0.06 \mathrm{MHz} / \mathrm{T}$, while approx. $0.8 \mathrm{MHz} / \mathrm{T}$ [34] or even $1000 \mathrm{MHz} / \mathrm{T}$ with the use of Love waves [35] are reported in the literature. The use of LNO-128Y cut can however easily increase the sensitivity by a factor of 3 . The field operation range in the current device is $100 \mathrm{mT}$, similar to what is observed in [34] (150-200 mT) and much wider than in [35] (1.5 mT).

This work mainly proves that the occurrence of strong stripes domains configurations in a perpendicularly magnetized material gives rise to reversible OOP response and is thus suitable for univocal OOP MSAW sensing.

\section{Conclusion}

As we have shown previously [7], the OOP magneto-acoustic response of magnetic SAW devices with IP easy magnetization is sensitive to the hysteretic behavior of IP magnetization components, which would limit the use of this geometry to build up an OOP field sensor.

In the search for alternative paths towards the development of OOP sensors, we have shown that the stabilization of an easy OOP magnetization with a strong stripes domains configuration (here in a $200 \mathrm{~nm}$ thick Ni based device) open interesting perspectives. The OOP magneto-acoustic response is indeed reversible, which is attributed to the specific magnetic configurations and reversal processes when stripe domains are stabilized. A bipolar response, i.e. a response that enables the measurement of both the field intensity and its sign, can thus be achieved. In-plane interrogations are however still hysteretic, which gives the $3 \mathrm{D}$ magnetic field detection in a single device rather limited. Further investigations are required to tailor the desirable magnetic anisotropy that could permit this $3 \mathrm{D}$ sensing ability. Beyond this promising proof of concept, another task is now to elaborate a strategy for increasing the amplitude of the frequency variation versus field, an essential prerequisite to obtain enough sensitivity and envision the development of sensors.

\section{Acknowledgements}

Experiments were carried out on IJL Project TUBE-Davm equipments funded by FEDER (EU), French PIA project "Lorraine Université d'Excellence" (ANR-15-IDEX-04LUE), Region Grand Est, Metropole Grand Nancy and ICEEL.

\section{References}

[1] Yamaguchi M, Hashimoto K Y, Kogo H, Naoe M 1980 IEEE. Trans. Magn. 16[2] Tiercelin N, Preobrazhensky V, Pernod P, Ostaschenko A 2008 Appl. Phys. Lett. 92062904 [3] Kadota M, Ito S, Ito Y, Hada T, Okaguchi K 2011 IEEE. International Ultrasonics Symposium Proceedings 
[4] Kadota M, Ito S, Ito Y, Hada T, Okaguchi K 2011 Jpn. J. Appl. Phys. 50 07HD07

[5] Webb D C, Forester D W, Ganguly A K, Vittoria C 1979 IEEE. Trans. Magn. 15

[6] Zhou H, Talbi A, Tiercelin N, Bou Matar O 2014 Appl. Phys. Lett. 104114101

[7] Polewczyk V, Dumesnil K, Lacour D, Moutaouekkil M, Mjahed H, Tiercelin N, Petit Watelot S, Dusch Y, Hage-Ali S, Elmazria O, Montaigne F, Talbi A, Bou Matar O, Hehn M 2017 Phys. Rev. App. 8024001

[8] Mishra H, Hehn M, Lacour D, Elmazria O, Tiercelin N, Mjahed H, Dumesnil K, Petit-Watelot S, Polewczyk V, Talbi A, Bou Matar O, Hage-Ali S 2019 Smart Materials and Structures. 108691

[9] Mathews S A, Bingham N S, Suess J R, Charipar K M, Auyeng A C Y, Kim Heugsoo, Charipar N A 2018 IEEE. Trans. On. Magn. $\mathbf{5 5} 2860936$

[10] Nicoloiu A, Ciubotaru F, Nastase C, Dinescu A, Iordanescu S, Ahmad H, Pirro P, Adelman C, Muller A 2019 20th International Conference on Solid-State Sensors, Actuators and Microsystems \& Eurosensors XXXIII 8808415 [11] Kittmann A, Durdaut P, Zabel S, Reermann J, Schmalz J, Spetzler B, Meyners D, Sun N X, McCord J, Gerken M, Schmidt G, Höft M, Knöchel R, Faupel F, Quandt E 2018 Sci. Rep. 8278

[12] Weiler M, Dreher L, Heeg C, Huebl H, Gross R, Brandt M S, Goennenwein S T B 2011 Phys. Rev. Lett. 106117601

[13] Weiler M, Huebl H, Goerg F S, Czeschka F D, Gross R, Goennenwein S T B 2012 Phys. Rev. Lett. 108176601

[14] Thevenard L, Gourdon C, Prieur J Y, Von Bardeleben H J, Vincent S, Becerra L, Largeau L, Duquesne J Y 2014 Phys. Rev. B. 90094401

[15] Thevenard L, Camara I S, Majrab S, Bernard M, Rovillain P, Lemaitre A, Gourdon C, Duquesne J Y 2016 Phys. Rev. B. 93134430

[16] Duquesne J Y, Rovillain P, Hepburn C, Eddrief M, Atkinson P, Anane A, Ranchal R, Marangolo M 2019 Phys. Rev. App. 12024042

[17] Verba R, Lisenkov I, Krivorotov I, Tiberkevich V, Slavin A 2018 Phys. Rev. App. 9064014

[18] Sasaki R, Nii Y, Iguchi, Onose Y 2017 Phys. Rev. B. 95 020407(R)

[19] Li X, Labanowski D, Salahuddin S, Lynch C S 2017 J. Appl. Phys. 122043904

[20] Polewczyk V 2018 PhD dissertation Université de Lorraine

[21] Malinowski G, Hehn M, Lenoble O, Schuhl A 2003 Phys. Rev. B. 68184404

[22] Danan H, Herr A, Meyer A J P 1968 J. Appl. Phys. 39669

[23] Hehn M, Ounadjela K, Ferré J, Grange W, Rousseaux F 1997 Appl. Phys. Lett. 712833

[24] Hehn M, Ounadjela K, Bucher J P, Rousseaux F, Decanini D, Bartenlijan B, Chappert C 1996 Science. 272 5269
[25] Hehn M, Padovani, Ounadjela K, Bucher J P 1996 Phys. Rev. B. 545

[26] Hehn M, Ounadjela K, Padovani S, Bucher J P, Arabski J, Bardou N, Bartenlian B, Chappert C, Rousseaux F, Decanini D, Carcenac F, Cambril E, Ravet M F 1996 J. of Appl. Phys. 795068

[27] Hehn M 1997 PhD dissertation Université Louis Pasteur [28] Hubert A, Schafer J J 1998 Magnetic domains: The analysis of magnetic microstructure Springer $\mathrm{p} 450$

[29] Marioni M A, Pilet N, Ashworth T V, O'Handley R C, Hug H J 2006 Phys. Rev. Lett. 97027201

[30] Ghidini M, Maccherozzi F, Moya X, Phillips L C, Yan W, Soussi J, Métallier N, Vickers M E, Steinke N J, Mansell R, Barnes C H W, Dhesi S S, Mathur N D 2015 Advanced Materials. 27 1460-1465

[31] Campbell C K 1998 Surface Acoustic Wave Devices for Mobile and Wireless Communications California: Academic Press

[32] Nazarchuk Z, Skalskyi V, Serhiyenko O 2017 Acoustic emission: Methodology and application Foundations of engineering mechanics

[33] Dreher L, Weiler M, Pernpeinter M, Huebl H, Gross R, Brandt M S, Goennenwein S T B 2012 Phys. Rev. B. 86 134415

[34] Elhosni M, Elmazria O, Petit-Watelot S, Bouvot L, Zhgoon S, Talbi A, Hehn M, Ait Aissa K, Hage-Ali S, Lacour D, Sarry F, Boumatar O 2016 Sens. Actu. A: Phys. 240 41-49 [35] Nan T, Hui Y, Rinaldi M, Sun N X 2013 Sci. Rep. 32013 